\title{
CURVA DE TOLERANCIA A LA GLUCOSA REFORZADA CON TRIAMCINOLONA EN EL ESTADO GRAVIDO-PUERPERAL
}

\section{Estudio preliminar}

\author{
Dr. Eduardo Acosta Bendeck 繁 \\ Dr. Néstor Vásquez Macías滋潾
}

Es un hecho que la medicina contemporánea debe proyectarse con la mayor energía, por razones que sobra enunciar aquí, hacia la medicina preventiva. Es éste un tema del que se han ocupado en todo tiempo los tratadistas e investigadores.

Uno de los grandes escritores clásicos españoles, aludía desde entonces al tema al poner en boca de uno de los personajes de "El Quijote" la siguiente expresión: No cura las enfermedades cuando las hay, sino que las previene para que no vengan".

Como es sabido en esta época de la medicina moderna existen métodos de gran precisión, que nos permiten hacer diagnósticos precoces en muchas enfermedades. Es así como se ha podido detectar prematuramente el trastorno metabólico latente de los glúcidos en pacientes prediabéticas en el estado grávido-puerperal. Lo anterior nos está permitiendo crear una conciencia más práctica y moderna del concepto que debe tener la medicina preventiva dentro del campo de la medicina actual.

E) trastorno metabólico latente de los glúcidos ha dado lugar a una variedad de denominaciones por diferen-

tes autores, prestándose a veces a confusiones en la terminología como son: prediabetes, diabetes latente, diabetes esencial, diabetes transitoria del embarazo, diabetes química, etc. términos todos estos que parecen corresponder a diferentes etapas evolutivas de una enfermedad genética determinada. Se acepta que toda paciente con diabetes tiene uno o más genes alterados; de acuerdo con las leyes de la herencia una persona es genética segura en los siguientes casos: en el gemalo univitelino de una diabética, o cuando ambos padres y abuelos son diabéticos; genética probable en los casos en los cuales hay un padre diabético o un abuelo diabético del otro padre, si éste es sano; también cuando hay dos abuelos diabéticos y un primo hermano con la enfermedad; y prediabética a toda paciente con antecedentes clínicos obstétricos que más adelante expondremos. Se conoce con el nombre de "Prediabetes" la etapa que va desde la concepción hasta cuando se ponga en evidencia la pri-

\footnotetext{
* Médico-Jefe del Dpto. de Obstetricia y Ginecología de la Clínica de Protección Familiar de Barranquilla.

** Médico Adjunto del Dpto. de Obstetricia y Ginecología de la Clínica de Protección Familiar de Barranquilla.
} 
mera anormalidad en la curva de tolerancia a la glucosa, es decir, en este estadio la curva de tolerancia a la glucosa standard o reforzada con corticoides son normales.

Antes de llegar a la etapa final del período prediabético, es decir, la diabetes clínica (aguda o crónica), hay una etapa intermedia que corresponde al periodo de la llamada "diabetes química".

Desde el punto de vista de la clínica, la polifagia, la polidipsia y la poliuria, han sido desplazados por los antecedentes clínicos obstétricos, como son el aumento de peso exagerado en el embarazo, muerte fetal anteparto, macrosomías, etc.

El estado prediabético es para el gineco-obstetra de especial interés ya que este período conlleva una serie de complicaciones en el embarazo y en el feto, aumentando la morbilidad materno-fetal, que son factibles de ser corregidas con un buen control y una terapia adecuada.

\section{Material y Métodos}

Se hizo el estudio en 111 pacientes, constituído por un primer grupo de 91 pacientes de la consulta prenatal de la Clínica de Protección Familiar de Barranquilla, seleccionadas por tener entre sus antecedentes clínicos obstétricos, tres o más de los siguientes signos: antecedentes familiares de diabetes, obesidad ( $10 \%$ de sobrepeso), aumento exagerado de peso durante el embarazo, óbitos, malformaciones congénitas, macrosomías, polihidramnios, toxemia, partos prematuros, abortos y vulvovaginitis o Cándida Albicans.

Se escogió un segundo grupo control de 20 pacientes gestantes sin antecedentes clínicos obstétricos de pre- diabetes, es decir, pacientes consideradas como normales.

Ambos grupos se estudiaron con curvas de tolerancia a la glucosa reforzadas con triamcinolona, esta última administrada en dosis de 8 mgrs. por dosis once y una hora antes de la prueba (administración de 100 grs. de glucosa por vía oral) en pacientes de menos de 66 kgrs. y 12 mgrs. por dosis en pacientes de más de 66 kgrs. Simultáneamente se practicó prueba de glucosa en orina. Ninguno de estos grupos se sometió a dieta especial previa a la prueba, debido a que en nuestro medio el contenido del régimen dietético es en un $80 \%$ a base de hidratos de carbono. Un número pequeño de pacientes se estudiaron con curvas simples.

Consideramos como positivas las pruebas, cuando dos o más parámetros se encontraron por encima de los valores siguientes: 115, 170, 135, 120, así: en ayunas, a la 1, 2 y 3 horas respectivamente. Fueron consideradas como sospechosas aquellas pacientes en las cuales se hallaba un parámetro alterado. Los valores de glicemia se tomaron por el método de Nelson-Somogyi. Estos valores normales de tolerancia a la glucosa reforzada con triamcinolona por vía oral fueron tomados del estudio realizado por Navarrete (5).

\section{Análisis de los Resultados}

El Cuadro 1 muestra 51 (56\%) pacientes prediabéticas estudiadas fuera del embarazo y 40 (44\%) estudiadas durante la gestación, en su mayoría en el segundo y tercer trimestre, ya que especialmente en estas últimas etapas del embarazo se produce una insuficiencia relativa de insulina por el aumento de algunas hormonas que neutralizan su efecto, como la hormona lactogénica, la somatotropina, los 
CUADRO NN 1

\begin{tabular}{lccccc}
\hline & \multicolumn{3}{c}{ Trimestre } & Total & $\%$ \\
& I & II & III & & \\
\hline Embarazadas & & 7 & 33 & 40 & 44 \\
No embarazadas & & & & 51 & 56 \\
\hline
\end{tabular}

corticoides, etc., aumentando la producción endógena de la glucosa, lo cual provoca una glucogénesis elevada, que es compensada por una glándula pancreática con función normal; pero cuando esta presenta alguna insuficiencia latente, se producen las alteraciones metabólicas de los glúcidos, debido a que no llega a cubrir la sobrecarga de glucosa endógena.

En el Cuadro 2, se señala que el mayor número de pacientes prediabéticas estudiadas correspondió a multíparas comprendidas entre las edades de 25 a 44 años. Las alteraciones metabólicas de los glúcidos se ponen de manifiesto a medida que la edad y paridad aumentan, según estudios de otros investigadores $(1,2,6)$. El promedio de edad de este grupo fue de 31.05 años.

\section{CUADRO № 2}

\begin{tabular}{lccrrr}
\hline Edad & No & \multicolumn{4}{c}{ Paridad } \\
& & nuli & primi & multi & gran m. \\
\hline $15-24$ & 18 & 1 & 15 & 4 & \\
$25-34$ & 39 & & 3 & 25 & 6 \\
$35-44$ & 34 & & 4 & 18 & 13 \\
48 & & & & 1 & 1 \\
TOTAL & 91 & 1 & 22 & 48 & 20 \\
\hline
\end{tabular}

En el Cuadro 3, observamos que los datos clínicos de obesidad, antecedentes familiares de diabetes, macrosomías, vulvovaginitis y abortos, fueron los signos clínicos de mayor frecuencia. Los mayores porcentajes de curvas alteradas se registraron en: macrosomías ( $52.6 \%$ ), antecedentes directo de diabetes $(51.7 \%)$, obesi- dad (51.6\%), óbitos $(43.3 \%)$ y polihidramnios $(40 \%)$ en orden de frecuencia.

\section{CUADRO N: 3}

\section{DATOS CLINICOS DE PREDIABETES}

\begin{tabular}{ll}
\hline & $\%$ \\
\hline Obesidad & 65,9 \\
Anteccdentes familiares de diabetes & 31,8 \\
Macrosomía & 23,7 \\
Vulvo vaginitis cándida albicans & 23 \\
Abortos & 17 \\
Obitos & 10 \\
Toxemia & 8,6 \\
Partos prematuros & 6,9 \\
Malformaciones & 1,8 \\
Polihidramnios & 1,3 \\
\hline
\end{tabular}

El Cuadro 4, corresponde al grupo contral con un total de 20 pacientes aparentemente normales, estudiadas durante el embarazo y en su mayoría en el último trimestre de la gestación.

\begin{tabular}{|c|c|c|c|c|}
\hline & & ADF & 4 & \\
\hline & & UPO & CONT & \\
\hline & Trimestre & & Total & $\%$ \\
\hline । & 11 & III & & \\
\hline 0 & 4 & 16 & 20 & 100 \\
\hline
\end{tabular}

En el Cuadro 5, vemos que el mayor número de casos estudiados en el grupo control, correspondió a primigestantes comprendidas entre las edades de 15 a 34 años. El promedio de edad fue de 24.4 años.

CUADRO № 5

GRUPO CONTROL

\begin{tabular}{lrccc}
\hline Edad & No & primi & $\begin{array}{l}\text { Paridad } \\
\text { multi }\end{array}$ & gran m. \\
\hline $15-24$ & 9 & 7 & 2 & \\
$25-34$ & 10 & 4 & 6 & \\
$35-44$ & 1 & & & 1 \\
TOTAL & 20 & 11 & 8 & 1 \\
\hline
\end{tabular}




\section{T G + TRIAMCINOLONA}

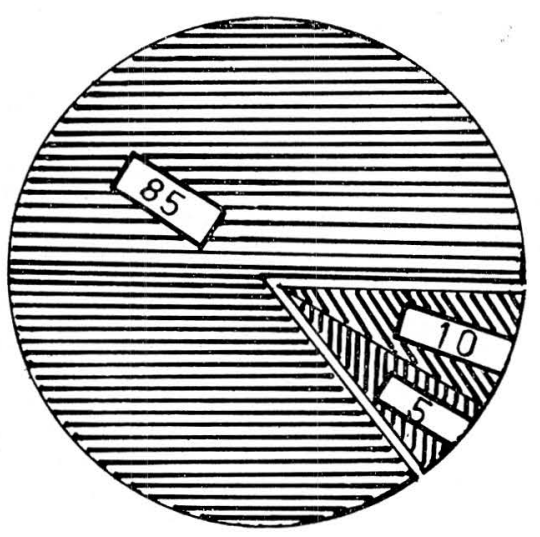

GRUPO CONTROL

\section{NORMALES SOSPECHOSAS ANORMALES

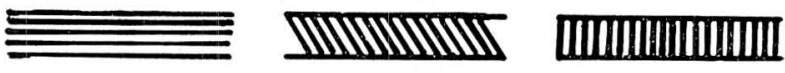

FIGURA N: 1

La Figura 1, corresponde al grupo control, en el cual el $85 \%$ fueron curvas normales, grupo éste que se clasificó como de pacientes normales; un $10 \%$ de curvas resultaron sospechosas y un $5 \%$ de curvas resultaron anormales, para un total de $15 \%$ de curvas alteradas, correspondientes a lo que se conoce con el nombre de diabetes química, en la cual sólo se registra una curva de tolerancia a la glucosa anormal y que se pone de presente en embarazos, estados infecciosos, stress o administración de corticoides. Tal parece que la diabetes química es un estado que precede a la diabetes clínica (4).

La Figura 2, muestra el resultado de curvas de tolerancia a la glucosa en el grupo de pacientes prediabéticas, estudiadas como tales y en el cual registramos un $54.9 \%$ de curvas nor- males, que corresponden a pacientes catalogadas como portadoras de un estado prediabético. Un $30.7 \%$ dieron curvas anormales o patológicas y que son consideradas como pacientes diabéticas y finalmente un $14.3 \%$ de curvas sospechosas, que corresponden a pacientes con un trastorno metabólico glúcido temprano. En términos generales se halló un $45 \%$ de curvas alteradas.

Finalmente el Cuadro 6 , es un análisis de diferentes autores acerca de la mortalidad perinatal en mujeres prediabéticas y tiene por objeto relievar la importancia del estado prediabético en la mujer embarazada en relación con el pronóstico fetal, objetivo de la obstetricia moderna. Se aprecia en el presente estudio una gran mortalidad perinatal y que aumenta cuando se le asocia alguna otra pato- 


\section{C.T.G + TRIAMCINOLONA}

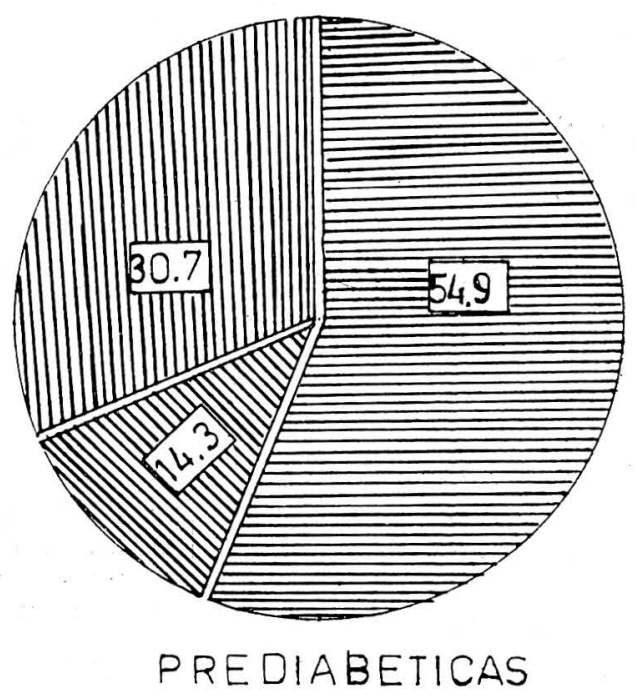

NORMALES SOSPECHOSAS ANORMALES
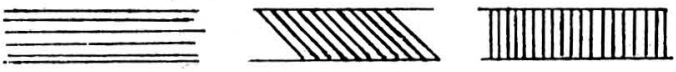

FIGURA № 2

logía. De aquí la importancia de detectar estos trastornos metabólicos en la mujer embarazada en una etapa temprana, con el fin de instalar una terapia precoz y adecuada (3).

\section{Comentarios}

Se lleva a cabo un estudio con curvas de tolerancia a la glucosa reforzadas con triamcinolona en $111 \mathrm{pa}-$ cientes en el estado grávido-puerperal; $54,9 \%$, se consideraron como prediabéticas, $30,7 \%$ diabéticas y $14,3 \%$ con trastorno metabólico glúcido temprano, resultando un total de $45 \%$ con alteraciones metabólicas de los glúcidos. La edad promedio fue de 31.05 años. En el grupo control, constituído por 20 pacientes, se registró un $85 \%$ de pacientes normales; un $10 \%$ con alteraciones metabólicas incipientes y un $5 \%$ con diabetes química. El estudio se hizo en la gran mayoría de los casos, en el último trimestre, que es considerado el más sensible para poner en evidencia un estado de diabetes latente.

Se escogió la traimcinolona por ser la demayor sensibilidad para descubrir más tempranamente a la futura diabética, como ha sido comprobado por otros investigadores. 


\section{CUADRO NN 6 \\ MORTALIDAD PERINATAL EN PREDIABETICAS}

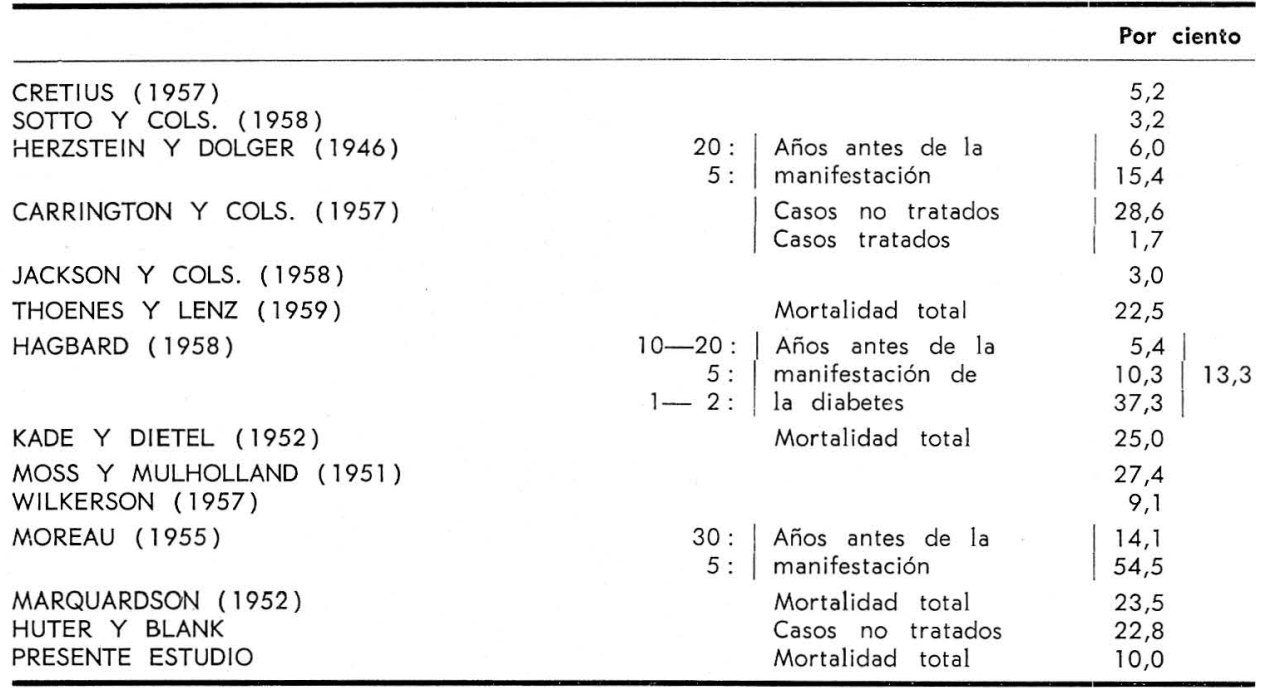

El embarazo es una excelente prueba funcional del sistema endocrino, ya que pone en evidencia una endocrinopatía de carácter hereditario, mucho antes de registrarse un trastorno metabólico de las grasas, carbohidratos y proteínas.

En el presente estudio se demuestra con claridad la importancia de la curva de tolerancia a la glucosa reforzada con triamcinolona en la investigación de la paciente prediabética en el estado grávido-puerperal. La importancia de esta prueba lo revela la gran mortalidad perinatal en este tipo de paciente no tratada.

\section{Resumen}

Un grupo seleccionado de pacientes sospechosas de diabetes, se estudia mediante la curva de tolerancia a la glucosa reforzada con Triamcinolona, que aparece como un método eficiente paar el diagnóstico temprano de la diabetes Mellitus.

\section{Summary}

A selected group of patients suspected with diabetes are studied through the curve of allowance of reinforced glucose with Triamcinolone; the latter seems to be an efficient method for early diagnosis of Mellitus diabetes.

\section{BIBLIOGRAFIA}

1 BOTELLA LLUSIA J.: Embriología endocrina prediabética o diabetes y gestación? Libro homenaje al Prof. Luis Castelazo Ayala. México. 1: 1813, 1970.

2 CARRINTON ELSIE R.: Diabetes in pregnancy. Clínica Obst. and Gynec. 16: 28, 1973.

3 HUTER VON A., BLANK H.: Significado,

Diagnóstico y Tratamiento de los estados prediabéticos en el embarazo. Sinopsis Obstétrico-Ginecología. 10: 1, 1963.

4 MOLINA B., 1: Desórdenes del Metabolismo Hidrocarbonado. Fundamentos de Medicina. 1: 289, 1970.

5 NAVARRETE V., y cols.: A Triamcinolone Glucose Tolerance Test in the Early Diagnosis of Diabetes. 14: 481, 1965.

6 VOTTA R. A., BAEZ J.: Algunas consideraciones sobre Diabetes e Infertilidad. Temas de Obstetricia y Ginecología. Libro homenaje al Dr. Alberto Duarte C. Ed. Stella. Bogotá. 1974. 